\title{
Triply heavy baryon spectroscopy in the relativistic quark model
}

\author{
R. N. Faustov $\odot$ and V. O. Galkin $\odot$ \\ Federal Research Center "Computer Science and Control", Russian Academy of Sciences, \\ Vavilov Street 40, 119333 Moscow, Russia
}

(Received 16 November 2021; accepted 3 January 2022; published 12 January 2022)

\begin{abstract}
Triply heavy baryons are investigated in the framework of the relativistic quark model based on the quark-diquark picture in the quasipotential approach in QCD. Masses of the ground and excited states of the $\Omega_{c c c}, \Omega_{b b b}, \Omega_{c c b}$, and $\Omega_{c b b}$ baryons are calculated. Orbital and radial excitations between the diquark and quark as well as between quarks inside the diquark are considered. The diquark internal structure is consistently taken into account by the form factor of the diquark-gluon interaction expressed through the overlap integral of the diquark wave functions. A detailed comparison with previous calculations is given.
\end{abstract}

DOI: 10.1103/PhysRevD.105.014013

\section{INTRODUCTION}

Recently, significant experimental progress has been achieved in studying hadrons with heavy quarks. Many new states of heavy mesons as well as of heavy baryons were observed, some of which have properties implying their exotic nature (for recent reviews, see, e.g., [1-3] and references therein). A special interest represents the longawaited discoveries very recently made by the $\mathrm{LHCb}$ Collaboration of the doubly charmed baryon $\Xi_{c c}^{++}$[4]; the tetraquark $T_{c c \bar{c} \bar{c}}$, composed of two charm quarks and two charm antiquarks, X(6900) [5]; and the doubly charmed tetraquark, $T_{c c}^{+}$, with a quark content $c c \bar{u} \bar{d}[6]$. All these new states require the production of at least two charm quark-antiquark pairs. The next important step forward will be the discovery of the triply heavy baryons, composed only from heavy charm and/or bottom quarks and, thus, requiring the production of three heavy quarkantiquark pairs. The first observation of the simultaneous production of three $J / \psi$ mesons in proton-proton collisions was very recently presented by the CMS Collaboration [7]. Estimates of the production cross section of triply heavy baryons in proton-proton [8] and heavy ion [9] collisions indicate that triply charmed $\Omega_{c c c}$ baryons have good chances to be observed at LHC.

In this paper, we apply the relativistic quark model based on the quasipotential approach in QCD to calculate the mass spectra of triply heavy baryons. These baryons contain only heavy quarks, and in the literature, they are usually treated as nonrelativistic systems. However, the

Published by the American Physical Society under the terms of the Creative Commons Attribution 4.0 International license. Further distribution of this work must maintain attribution to the author(s) and the published article's title, journal citation, and DOI. Funded by SCOAP. investigation of the heavy quark dynamics in heavy quarkonia shows that heavy quarks should be treated relativistically [10]. Indeed, estimates of the charm quark velocity $v$ in charmonia show that it is about one half of the velocity of light $c$, while the bottom quark velocity in bottomonia is about one third of $c$. Our previous investigations of meson [10,11], baryon [12-15], and tetraquark $[3,16]$ properties showed that relativistic effects play a very important role. Thus, we treat triply heavy baryons completely relativistically without application of the expansion in heavy quark velocity. To achieve this goal, we use the relativistic quark-diquark model which was previously developed and applied for the consideration of heavy [12,13], doubly heavy [14], and strange [15] baryons. Constructing the triply heavy baryon, we assume that two quarks of the same flavor form a doubly heavy diquark, ${ }^{1}$ and the baryon is a relativistic bound system of this doubly heavy diquark and heavy quark. The masses and wave functions of diquarks are obtained by solving the relativistic quasipotential equation with the quark-quark interaction, which is one half of the quark-antiquark interaction in mesons. The diquark is considered to be a composite, not a pointlike object. The diquark internal structure is taken into account by calculating the diquark form factor, which enters the diquark-gluon interaction, and is expressed as the overlap integral of the diquark wave functions. The account of the diquark size softens the diquark-gluon interaction thus increasing the baryon mass. This effect allowed us to get the correct prediction for the doubly charmed baryon $\Xi_{c c}^{++}$mass long before its discovery [14]. It is important to point out that consistent treatment of

\footnotetext{
${ }^{1}$ This assumption was previously successfully tested in calculations of strange baryon spectra [15]. All observed ground and excited states of $\Lambda, \Sigma$, and $\Xi$ baryons fit well in the picture where diquarks are composed from quarks of the same constituent mass.
} 
the relativistic quark dynamics permitted us to get predictions for meson [10,11], baryon [12-15], and tetraquark [3] masses and decays in good agreement with experimental data using the universal set of model parameters, which we keep fixed in the present calculations of the triply baryon spectroscopy. Note that in most quark models the parameters for descriptions of meson and baryon properties are varied.

The paper is organized as follows. In Sec. II, we briefly describe our relativistic quark-diquark model. The quasipotential equation and quark-quark and quark-diquark interaction potentials are given. Doubly heavy diquarks are considered in Sec. III. Their masses are calculated up to the first radial excitation and second orbital excitation. The form factors entering the diquark-gluon interaction are evaluated, and their appropriate parametrization is given. In Sec. IV, we calculate the masses of the ground, orbitally, and radially excited states of triply heavy baryons and compare our results with previous calculations. Finally, Sec. V contains our conclusions.

\section{RELATIVISTIC QUARK-DIQUARK MODEL}

The relativistic quark-diquark model for the description of doubly heavy, heavy baryon, and hyperon spectroscopy based on the quasipotential approach and quark-diquark picture of baryons was developed and previously used in Refs. [12-15]. Here, we apply this model for the consideration of the triply heavy baryon spectroscopy. We use the same assumptions and model parameters. For completeness, we give its brief outline. In this approach, the complicated relativistic three-body problem is reduced to the solution of two more simple relativistic two-body problems. First, we introduce diquarks which are considered to be bound states of two quarks. It is assumed that quarks of the same flavor form a diquark. Second, the baryon is considered to be a bound system of a diquark and quark. In a quasipotential approach, interactions of two quarks in a diquark and of the quark and diquark in a baryon are described by the diquark wave function $\Psi_{d}$ and by the baryon wave function $\Psi_{B}$, respectively. These wave functions satisfy the quasipotential equation of the Schrödinger type [10]

$$
\left(\frac{b^{2}(M)}{2 \mu_{R}}-\frac{\mathbf{p}^{2}}{2 \mu_{R}}\right) \Psi_{d, B}(\mathbf{p})=\int \frac{d^{3} q}{(2 \pi)^{3}} V(\mathbf{p}, \mathbf{q} ; M) \Psi_{d, B}(\mathbf{q}),
$$

with the relativistic reduced mass given by

$$
\mu_{R}=\frac{M^{4}-\left(m_{1}^{2}-m_{2}^{2}\right)^{2}}{4 M^{3}},
$$

and the center-of-mass system relative momentum squared on a mass shell defined by

$$
b^{2}(M)=\frac{\left[M^{2}-\left(m_{1}+m_{2}\right)^{2}\right]\left[M^{2}-\left(m_{1}-m_{2}\right)^{2}\right]}{4 M^{2}},
$$

where $M$ is the bound state mass (diquark or baryon), $m_{1,2}$ are the masses of quarks $\left(q_{1}\right.$ and $\left.q_{2}\right)$ which form the diquark or of the diquark $(d)$ and quark $(q)$ which form the baryon $(B)$, and $\mathbf{p}$ is their relative momentum.

To construct the kernel $V(\mathbf{p}, \mathbf{q} ; M)$ in Eq. (1), which is the quasipotential operator of the quark-quark or quark-diquark interactions, it is assumed that the effective interaction is the sum of the usual one-gluon exchange term and the mixture of long-range vector and scalar linear confining potentials $[12,13]$. The vector confining potentials contain additional effective Pauli terms, which introduce anomalous chromomagnetic moments of quarks and diquarks.

The quark-quark $(q q)$ interaction quasipotential for the diquark is given by

$V(\mathbf{p}, \mathbf{q} ; M)=\bar{u}_{1}(p) \bar{u}_{2}(-p) \mathcal{V}(\mathbf{p}, \mathbf{q} ; M) u_{1}(q) u_{2}(-q)$,

with

$$
\begin{aligned}
\mathcal{V}(\mathbf{p}, \mathbf{q} ; M)= & \frac{1}{2}\left[\frac{4}{3} \alpha_{s} D_{\mu \nu}(\mathbf{k}) \gamma_{1}^{\mu} \gamma_{2}^{\nu}+V_{\text {conf }}^{V}(\mathbf{k}) \Gamma_{1}^{\mu}(\mathbf{k}) \Gamma_{2 ; \mu}(-\mathbf{k})\right. \\
& \left.+V_{\text {conf }}^{S}(\mathbf{k})\right],
\end{aligned}
$$

where $\alpha_{s}$ is the QCD coupling constant, $D_{\mu \nu}$ is the gluon propagator in the Coulomb gauge, $\mathbf{k}=\mathbf{p}-\mathbf{q}, \gamma_{\mu}$, and $u(p)$ are the Dirac matrices and spinors. The effective long-range vector vertex of the quark is defined by [10]

$$
\Gamma_{\mu}(\mathbf{k})=\gamma_{\mu}+\frac{i \kappa}{2 m} \sigma_{\mu \nu} \tilde{k}^{\nu}, \quad \tilde{k}=(0, \mathbf{k}),
$$

and $\kappa$ is the anomalous chromomagnetic moment of quarks.

The quark-diquark $(q d)$ interaction quasipotential in the baryon has the form

$$
\begin{aligned}
V(\mathbf{p}, \mathbf{q} ; M)= & \frac{\left\langle d(P)\left|J_{\mu}\right| d(Q)\right\rangle}{2 \sqrt{E_{d}(p) E_{d}(q)}} \bar{u}_{q}(p) \frac{4}{3} \alpha_{s} D_{\mu \nu}(\mathbf{k}) \gamma^{\nu} u_{q}(q) \\
& +\psi_{d}^{*}(P) \bar{u}_{q}(p) J_{d ; \mu} \Gamma_{q}^{\mu}(\mathbf{k}) V_{\mathrm{conf}}^{V}(\mathbf{k}) u_{q}(q) \psi_{d}(Q) \\
& +\psi_{d}^{*}(P) \bar{u}_{q}(p) V_{\mathrm{conf}}^{S}(\mathbf{k}) u_{q}(q) \psi_{d}(Q)
\end{aligned}
$$

where $\left\langle d(P)\left|J_{\mu}\right| d(Q)\right\rangle$ is the vertex of the diquark-gluon interaction which takes into account the diquark internal structure, $J_{d ; \mu}$ is the effective long-range vector vertex of the diquark, the diquark momenta are $P=\left(E_{d}(p),-\mathbf{p}\right)$, $Q=\left(E_{d}(q),-\mathbf{q}\right)$ with $E_{d}(p)=\sqrt{\mathbf{p}^{2}+M_{d}^{2}}$, and $\psi_{d}(P)$ is the diquark wave function [12].

The vector and scalar confining potentials in the nonrelativistic limit in configuration space are the linear potentials with the mixing coefficient $\varepsilon$, 
$V_{\text {conf }}^{V}(r)=(1-\varepsilon)(A r+B), \quad V_{\text {conf }}^{S}(r)=\varepsilon(A r+B)$,

and their sum is just the usual static Cornell-like potential,

$$
V(r)=-\frac{4}{3} \frac{\alpha_{s}}{r}+A r+B
$$

with the freezing QCD coupling constant

$$
\alpha_{s}\left(\mu^{2}\right)=\frac{4 \pi}{\left(11-\frac{2}{3} n_{f}\right) \ln \frac{\mu^{2}+M_{B}^{2}}{\Lambda^{2}}} .
$$

The scale $\mu=2 \mu_{N R}$ is chosen to be twice the nonrelativistic reduced mass $\mu_{N R}=m_{1} m_{2} /\left(m_{1}+m_{2}\right)$. The background mass is taken $M_{B}=0.95 \mathrm{GeV}$, and the parameter $\Lambda=$ $413 \mathrm{MeV}$ was fixed from the light meson spectroscopy [11].

All parameters of the model are kept fixed from previous calculations of meson and baryon properties $[10,12,14]$. The constituent heavy quark masses are $m_{c}=1.55 \mathrm{GeV}$, $m_{b}=4.88 \mathrm{GeV}$. The parameters of the linear potential are $A=0.18 \mathrm{GeV}^{2}$ and $B=-0.3 \mathrm{GeV}$. The value of the mixing coefficient of vector and scalar confining potentials is $\varepsilon=-1$, and the anomalous chromomagnetic quark moment is $\kappa=-1$. Note that the long-range chromomagnetic contribution to the potential, which is proportional to $(1+\kappa)$, vanishes for the chosen value of $\kappa=-1$.

\section{DOUBLY HEAVY DIQUARKS}

As the first step, we consider the doubly heavy diquarks and calculate their masses and form factors. We assume that in triply heavy baryons two quarks of the same flavor form a doubly heavy diquark. We solve the quasipotential equation (1) numerically with the complete relativistic potential (4). Since a diquark is composed of heavy quarks of the same flavor, it is necessary to take into account the Pauli principle. The total baryon wave function must be antisymmetric. It is antisymmetric in color; thus, the rest of the wave function must be symmetric. The symmetric form of the flavor part of the considered doubly heavy diquark implies that the product of the spin and orbital parts is also symmetric. For the $S$ and $D$ states, which have orbitally symmetric wave functions, the diquark spin wave function must be also symmetric, and thus, the diquark spin is 1 . For the $P$ states with antisymmetric orbital wave function, the diquark spin wave function must be also antisymmetric, and the diquark spin is 0 . The resulting value of the total momentum of the diquark is $j=1$ for the $S$ and $P$ states, while values $j=1,2,3$ are possible for the $D$ states. Note that we do not consider higher orbital excitations of the quarks inside the diquark.

The calculated masses of the ground and excited states of diquarks are presented in Table I. We also give the values of the parameters $\xi$ and $\zeta$. They parametrize with high accuracy the $r$ dependence of the form factor $F(r)$ in
TABLE I. Masses $M$ and form factor parameters of the doubly heavy diquarks.

\begin{tabular}{lcccc}
\hline \hline Quark content & State $n l_{j}$ & $M(\mathrm{MeV})$ & $\xi(\mathrm{GeV})$ & $\zeta\left(\mathrm{GeV}^{2}\right)$ \\
\hline$c c$ & $1 s_{1}$ & 3226 & 1.30 & 0.42 \\
& $1 p_{1}$ & 3460 & 0.74 & 0.315 \\
& $2 s_{1}$ & 3535 & 0.67 & 0.19 \\
& $1 d_{1,2,3}$ & 3704 & 0.39 & 0.42 \\
& $2 p_{1}$ & 3712 & 0.60 & 0.155 \\
$b b$ & $1 s_{1}$ & 9778 & 1.30 & 1.60 \\
& $1 p_{1}$ & 9944 & 0.90 & 0.59 \\
& $2 s_{1}$ & 10015 & 0.85 & 0.31 \\
& $1 d_{1,2,3}$ & 10123 & 0.49 & 0.59 \\
& $2 p_{1}$ & 10132 & 0.65 & 0.215 \\
\hline \hline
\end{tabular}

the vertex of the diquark-gluon interaction (6), which is calculated through the overlap integral of the diquark wave functions [12]. It is expressed by

$$
F(r)=1-e^{-\xi r-\zeta r^{2}}
$$

and takes the internal structure of a diquark into account [12] smearing the diquark-gluon interaction. In this table, we use the lowercase letters to denote diquark quantum numbers. This is done to distinguish them from the quarkdiquark excitations for which we reserve the uppercase letters. Here, $n=n_{r}+1$, where $n_{r}$ is the radial quantum number (the number of nodes of the wave function); $l=$ $s, p, d \ldots$ is the orbital momentum and $j$ the total momentum of the diquark. The calculations show that the masses of the $d$ states with $j=1,2,3$ differ by less than $1 \mathrm{MeV}$; thus, we consider their masses to be equal and give only one value.

\section{TRIPLY HEAVY BARYONS}

As the second step, we calculate the masses of the triply heavy baryons as the bound states of a heavy quark and doubly heavy diquark. Evaluating the baryon masses, we treat all relativistic contributions nonperturbatively. The quark-diquark quasipotential contains the relativistic contributions both to the spin-independent $V_{\mathrm{SI}}$ and spindependent $V_{\mathrm{SD}}$ parts

$$
V(r)=V_{\mathrm{SI}}(r)+V_{\mathrm{SD}}(r) .
$$

The spin-independent part determines the position of centers of gravity of the baryon levels, while the spindependent part is responsible for their fine and hyperfine splittings. These parts are expressed through the static potential and its derivatives. The explicit expressions for these potentials can be found in Refs. [12,13]. It is important to point out that, as it was already noted in the previous section, the diquark form factor $F(r)$ smears the diquark-gluon interaction, thus accounting for its 
internal structure. As a result, in the nonrelativistic limit, the one-gluon exchange part of the quark-diquark potential is modified and has the form of the smeared Coulomb-like potential

$$
\hat{V}_{\text {Coul }}(r)=-\frac{4}{3} \alpha_{s} \frac{F(r)}{r},
$$

with $F(r)$ given by Eq. (10).

The spin-dependent part of the quasipotential contains the spin-orbit, tensor, and spin-spin interactions. It has the following form [13]:

$$
\begin{aligned}
V_{\mathrm{SD}}(r)= & a_{1} \mathbf{L S}_{d}+a_{2} \mathbf{L} \mathbf{S}_{Q} \\
& +b\left[-\mathbf{S}_{d} \mathbf{S}_{Q}+\frac{3}{r^{2}}\left(\mathbf{S}_{d} \mathbf{r}\right)\left(\mathbf{S}_{Q} \mathbf{r}\right)\right]+c \mathbf{S}_{d} \mathbf{S}_{Q},
\end{aligned}
$$

where $\mathbf{L}$ is the orbital angular momentum; $\mathbf{S}_{d}$ and $\mathbf{S}_{Q}$ are the diquark and quark spin operators, respectively. The coefficients $a_{1}, a_{2}, b$, and $c$ are expressed through the corresponding derivatives of the smeared Coulomb and confining potentials [13]. The smearing of the one-gluon exchange potential (12) naturally softens singularities in the relativistic quasipotential in configuration space and allows us to solve numerically the quasipotential equation in its complete relativistic form. Note that both the one-gluon exchange and confining potentials contribute to the quarkdiquark spin-orbit interaction. The presence of the spinorbit $\mathbf{L} \mathbf{S}_{Q}$ and of tensor terms in the quark-diquark potential leads to a mixing of states with the same total angular momentum $J$ and parity $P$ but different values of the diquark angular momentum $\left(\mathbf{L}+\mathbf{S}_{d}\right)$. We consider such mixing in the same way as in the case of doubly heavy baryons [14].

We calculate masses of all triply heavy baryons: $\Omega_{c c c}$, $\Omega_{b b b}, \Omega_{c c b}$, and $\Omega_{c b b}$. Their calculated spectra are given in Tables II-V. In the left hand half of these tables, we give

TABLE II. Masses of the $\Omega_{c c c}$ states.

\begin{tabular}{cccccc}
\hline \hline$J^{P}$ & $N L n l$ & Mass $(\mathrm{MeV})$ & $J^{P}$ & $N L n l$ & Mass $(\mathrm{MeV})$ \\
\hline$\frac{1}{2}^{+}$ & $2 S 1 s_{-}, 1 P 1 p$ & 5230 & $\frac{1}{2}{ }^{-}$ & $1 S 1 p, 1 P 1 s$ & 5010 \\
& $1 D 1 s$ & 5278 & & $1 P 2 s_{-}, 2 S 1 p_{-}$ & 5370 \\
$\frac{3}{2}^{+}$ & $1 S 1 s$ & 4712 & & $2 S 1 p_{+}$ & 5385 \\
& $1 S 2 s_{+}$ & 5137 & & $1 D 1 p_{+}$ & 5520 \\
& $1 D 1 s_{+}$ & 5267 & $\overline{3}^{-}$ & $1 S 1 p, 1 P 1 s$ & 5029 \\
& $1 D 1 s_{-}, 1 P 1 p$ & 5277 & & $1 P 2 s_{-}, 2 S 1 p_{-}$ & 5379 \\
& $2 S 2 s$ & 5541 & & $2 S 1 p_{+}$ & 5394 \\
${ }_{5}{ }^{+}$ & $1 D 1 s_{-}, 1 P 1 p$ & 5278 & & $1 D 1 p_{+}$ & 5522 \\
& $1 D 1 s_{+}$ & 5290 & $\frac{5}{2}-$ & $1 F 1 s$ & 5519 \\
$\frac{7}{2}+$ & $1 D 1 s_{+}$ & 5291 & & $1 P 1 d_{+}$ & 5523 \\
& & & $\frac{7}{2}-$ & $1 F 1 s$ & 5517 \\
& & & & $1 P 1 d_{+}$ & 5526 \\
\hline \hline
\end{tabular}

TABLE III. Masses of the $\Omega_{b b b}$ states.

\begin{tabular}{|c|c|c|c|c|c|}
\hline$J^{P}$ & $N L n l$ & Mass $(\mathrm{MeV})$ & $J^{P}$ & $N L n l$ & Mass $(\mathrm{MeV})$ \\
\hline \multirow[t]{7}{*}{$\frac{1}{2}+$} & $1 S 1 s$ & 7984 & \multirow{7}{*}{$\frac{1}{2}^{-}$} & $1 P 1 s$ & 8250 \\
\hline & $1 S 2 s$ & 8361 & & $1 S 1 p$ & 8266 \\
\hline & $2 S 1 s$ & 8405 & & $1 P 1 s$ & 8268 \\
\hline & $1 D 1 s$ & 8472 & & $1 S 2 p$ & 8550 \\
\hline & $1 P 1 p$ & 8505 & & $2 P 1 s$ & 8583 \\
\hline & $1 P 1 p$ & 8511 & & $2 P 1 s$ & 8591 \\
\hline & $1 S 1 d$ & 8531 & & $1 P 2 s$ & 8592 \\
\hline \multirow[t]{8}{*}{$\frac{3}{2}+$} & $1 S 1 s$ & 7999 & \multirow{8}{*}{$\frac{3}{2}-$} & $1 P 2 s$ & 8595 \\
\hline & $1 S 2 s$ & 8366 & & $1 P 1 s$ & 8262 \\
\hline & $2 S 1 s$ & 8412 & & $1 P 1 s$ & 8268 \\
\hline & $1 D 1 s$ & 8474 & & $1 S 1 p$ & 8273 \\
\hline & $1 D 1 s$ & 8476 & & $1 S 2 p$ & 8554 \\
\hline & $1 P 1 p$ & 8506 & & $2 P 1 s$ & 8587 \\
\hline & $1 P 1 p$ & 8510 & & $2 P 1 s$ & 8591 \\
\hline & $1 S 1 d$ & 8534 & & $1 P 2 s$ & 8591 \\
\hline \multirow[t]{4}{*}{$\frac{5}{2}^{+}$} & $1 D 1 s$ & 8473 & \multirow{4}{*}{$\frac{5}{2}^{-}$} & $1 P 2 s$ & 8594 \\
\hline & $1 D 1 s$ & 8476 & & $1 P 1 s$ & 8267 \\
\hline & $1 P 1 p$ & 8508 & & $2 P 1 s$ & 8590 \\
\hline & $1 S 1 d$ & 8536 & & $1 P 2 s$ & 8592 \\
\hline \multirow[t]{2}{*}{$\frac{7}{2}+$} & $1 D 1 s$ & 8473 & \multirow[t]{2}{*}{$\frac{7}{2}^{-}$} & \multirow[t]{2}{*}{$1 F 1 s$} & \multirow[t]{2}{*}{8647} \\
\hline & $1 S 1 d$ & 8538 & & & \\
\hline
\end{tabular}

\begin{tabular}{cccccc}
\hline \hline$J^{P}$ & $N L n l$ & Mass $(\mathrm{MeV})$ & $J^{P}$ & $N L n l$ & Mass $(\mathrm{MeV})$ \\
\hline$\frac{1}{2}^{+}$ & $2 S 1 s_{-}, 1 P 1 p$ & 14877 & $\frac{1}{2}^{-}$ & $1 S 1 p, 1 P 1 s$ & 14698 \\
& $1 D 1 s$ & 14912 & & $1 P 2 s_{-}, 2 S 1 p_{-}$ & 14991 \\
$\frac{3}{2}^{+}$ & $1 S 1 s$ & 14468 & & $2 S 1 p_{+}$ & 15042 \\
& $1 S 2 s_{+}$ & 14815 & & $1 D 1 p_{+}$ & 15088 \\
& $1 D 1 s_{-}, 1 P 1 p$ & 14893 & $\overline{3}^{-}$ & $1 S 1 p, 1 P 1 s$ & 14702 \\
& $1 D 1 s_{+}$ & 14905 & & $1 P 2 s_{-}, 2 S 1 p_{-}$ & 14922 \\
& $2 S 2 s$ & 15123 & & $2 S 1 p_{+}$ & 15031 \\
$\frac{5}{2}^{+}$ & $1 D 1 s_{-}, 1 P 1 p$ & 14895 & & $1 D 1 p_{+}$ & 15089 \\
& $1 D 1 s_{+}$ & 14907 & $\frac{5}{2}-$ & $1 F 1 s$ & 15081 \\
$\frac{7}{2}^{+}$ & $1 D 1 s_{+}$ & 14909 & & $1 P 1 d_{+}$ & 15086 \\
& & & $\frac{7}{2}$ & $1 F 1 s$ & 15082 \\
& & & & $1 P 1 d_{+}$ & 15089 \\
\hline \hline
\end{tabular}

TABLE IV. Masses of the $\Omega_{c c b}$ states.

states with the positive parity and in the right one the negative parity. We use the standard notations for the baryon states $J^{P}$, where $J$ and $P$ are the baryon total spin and parity, respectively. The composition of the baryon state is given by $N L n l_{j}$, where the capital letters denote quantum numbers of the quark-diquark system and the lowercase letters the diquark state. $N$ or $n$ is the radial quantum number (the number of nodes of the wave function) plus one, and $L$ or $l$ is the orbital quantum number.

For the baryons composed of identical quarks $\left(\Omega_{c c c}\right.$ and $\Omega_{b b b}$ ), there is an additional complication. It is necessary to 
TABLE V. Masses of the $\Omega_{c b b}$ states.

\begin{tabular}{|c|c|c|c|c|c|}
\hline$J^{P}$ & $N L n l$ & Mass $(\mathrm{MeV})$ & $J^{P}$ & $N L n l$ & Mass $(\mathrm{MeV})$ \\
\hline \multirow[t]{7}{*}{$\frac{1}{2}^{+}$} & $1 S 1 s$ & 11198 & \multirow{7}{*}{$\frac{1}{2}^{-}$} & $1 P 1 s$ & 11414 \\
\hline & $1 S 2 s$ & 11507 & & $1 S 1 p$ & 11506 \\
\hline & $1 S 1 d$ & 11622 & & $1 P 1 s$ & 11540 \\
\hline & $2 S 1 s$ & 11690 & & $1 S 2 p$ & 11654 \\
\hline & $1 P 1 p$ & 11692 & & $1 P 2 s$ & 11778 \\
\hline & $1 P 1 p$ & 11714 & & $1 P 2 s$ & 11796 \\
\hline & $1 D 1 s$ & 11796 & & $2 S 1 p$ & 11893 \\
\hline \multirow[t]{8}{*}{$\frac{3}{2}+$} & $1 S 1 s$ & 11217 & \multirow[t]{7}{*}{$\frac{3}{2}-$} & $1 S 1 p$ & 11424 \\
\hline & $1 S 2 s$ & 11515 & & $1 P 1 s$ & 11535 \\
\hline & $1 S 1 d$ & 11629 & & $1 P 1 s$ & 11541 \\
\hline & $2 S 1 s$ & 11700 & & $1 S 2 p$ & 11660 \\
\hline & $1 P 1 p$ & 11707 & & $1 P 2 s$ & 11788 \\
\hline & $1 P 1 p$ & 11717 & & $1 P 2 s$ & 11795 \\
\hline & $1 D 1 s$ & 11797 & & $2 S 1 p$ & 11897 \\
\hline & $1 D 1 s$ & 11807 & \multirow{2}{*}{$\frac{5}{2}-$} & $1 P 1 s$ & 11543 \\
\hline \multirow[t]{4}{*}{$\frac{5}{2}^{+}$} & $1 S 1 d$ & 11632 & & $1 P 2 s$ & 11795 \\
\hline & $1 P 1 p$ & 11715 & \multirow[t]{3}{*}{$\frac{7-}{2}$} & $1 P 1 d$ & 11903 \\
\hline & $1 D 1 s$ & 11806 & & & \\
\hline & $1 D 1 s$ & 11807 & & & \\
\hline$\underline{\frac{7}{2}+}$ & $1 S 1 d$ & 11635 & & & \\
\hline
\end{tabular}

take into account the Pauli principle not only for the diquark but also for the entire baryon. It requires the total wave function to be antisymmetric. The color wave function of the baryon is antisymmetric. The flavor part is symmetric. This means that the spin-momentum part of the wave function must be fully symmetric. For the ground $1 S 1 s$ state, this part is symmetric in momentum, and thus, the spin wave functions must be also fully symmetric, which corresponds to the total baryon spin $3 / 2$. Therefore, only the $3 / 2^{+}$ ground state is possible. The lightest $1 / 2^{+}$state should contain excitations and has a significantly larger mass. The excited states are combinations of orbital and/or radial excitations of the diquark and/or quark-diquark bound systems with the fully symmetric (3/2) or mixed symmetry $(1 / 2)$ spin wave functions. Symmetric combinations, such as, e.g., $\quad|2 S 1 s\rangle_{+}=(|2 S 1 s\rangle+|1 S 2 s\rangle) / \sqrt{2}, \quad|1 D 1 s\rangle_{+}=$ $(|1 D 1 s\rangle+|1 S 1 d\rangle) / \sqrt{2}, \quad|2 S 2 s\rangle$, are combined with a fully symmetric spin $3 / 2$ wave function. While antisymmetric combinations, such as, e.g., $|2 S 1 s\rangle_{-}=\left(|2 S 1 s\rangle_{-}\right.$ $|1 S 2 s\rangle) / \sqrt{2},|1 D 1 s\rangle_{-}=(|1 D 1 s\rangle-|1 S 1 d\rangle) / \sqrt{2},|1 P 2 s\rangle_{-}=$ $(|1 P 2 s\rangle-|1 S 2 p\rangle) / \sqrt{2}$, are combined with the mixed symmetry spin $1 / 2$ wave functions. The details can be found in Ref. [17].

Masses of the ground states of $\Omega_{c c c}, \Omega_{b b b}, \Omega_{c c b}$, and $\Omega_{c b b}$ baryons were calculated in many papers based on different approaches [17-46]. The predicted masses of the ground state $3 / 2^{+} \Omega_{c c c}$ baryons range from 4670 to $4990 \mathrm{MeV}$, and masses of the $3 / 2^{+} \Omega_{b b b}$ baryons range from 13280 to $14834 \mathrm{MeV}$. Our predictions for these masses, $4712 \mathrm{MeV}$ and $14468 \mathrm{MeV}$, respectively, are well inside both ranges.

Excited states received significantly less attention. In Tables VI-IX, we compare our predictions with previous calculations [17-28] for the masses of $\Omega_{c c c}, \Omega_{b b b}, \Omega_{c c b}$, and $\Omega_{c b b}$ baryons. Masses of the triply heavy baryons were calculated using lattice QCD with dynamical light quark

TABLE VI. Comparison with previous theoretical predictions for the masses of the $\Omega_{c c c}$ states.

\begin{tabular}{|c|c|c|c|c|c|c|c|c|}
\hline$J^{P}$ & Our (MeV) & [18] (MeV) & [19] (MeV) & [17] $(\mathrm{MeV})$ & [20] $(\mathrm{MeV})$ & [21] $(\mathrm{MeV})$ & [22] $(\mathrm{MeV})$ & [23] (MeV) \\
\hline \multirow{2}{*}{$\frac{1}{2}+$} & 5230 & $5397(13)$ & 5376 & 5358 & 5473 & 5325 & 5352 & \\
\hline & 5278 & $5403(14)$ & & & & 5332 & 5373 & \\
\hline \multirow{4}{*}{$\frac{3}{2}+$} & 4712 & $4761(6)$ & 4798 & 4797 & 4806 & 4965 & 4828 & 4760 \\
\hline & 5137 & $5315(31)$ & 5286 & 5309 & 5300 & 5313 & 5285 & 5150 \\
\hline & 5267 & $5428(13)$ & 5376 & 5358 & 5448 & & 5368 & \\
\hline & 5277 & $5463(13)$ & & & & & 5412 & \\
\hline \multirow{2}{*}{$\frac{5}{2}+$} & 5278 & $5404(15)$ & 5376 & 5358 & 5416 & 5329 & 5392 & \\
\hline & 5290 & $5462(15)$ & & & & 5343 & 5433 & \\
\hline$\frac{7}{2}^{+}$ & 5291 & $5395(49)$ & 5376 & 5358 & 5375 & 5331 & 5418 & \\
\hline \multirow{3}{*}{$\frac{1-}{2}$} & 5010 & $5118(9)$ & 5129 & 5103 & 5012 & 5155 & 5142 & \\
\hline & 5370 & $5610(31)$ & 5525 & & 5607 & & & \\
\hline & 5385 & $5629(43)$ & & & & & & \\
\hline \multirow{3}{*}{$\frac{3}{2}-$} & 5029 & $5122(13)$ & 5129 & 5103 & 4991 & 5160 & 5162 & 5027 \\
\hline & 5379 & $5660(31)$ & 5525 & & 5584 & & & \\
\hline & 5394 & $5722(44)$ & & & & & & \\
\hline \multirow[t]{2}{*}{$\frac{5-}{2}$} & 5519 & $5514(64)$ & 5558 & & 4965 & & & \\
\hline & 5523 & $5707(25)$ & & & 5584 & & & \\
\hline$\frac{7-}{2}$ & 5517 & $5679(28)$ & & & 5829 & & & \\
\hline
\end{tabular}


TABLE VII. Comparison with previous theoretical predictions for the masses of the $\Omega_{b b b}$ states.

\begin{tabular}{|c|c|c|c|c|c|c|c|c|}
\hline$J^{P}$ & Our $(\mathrm{MeV})$ & [24] (MeV) & [19] (MeV) & [17] $(\mathrm{MeV})$ & [20] $(\mathrm{MeV})$ & [21] $(\mathrm{MeV})$ & [22] $(\mathrm{MeV})$ & [23] (MeV) \\
\hline \multirow{2}{*}{$\frac{1}{2}^{+}$} & 14877 & $14938(18)$ & 14894 & 14896 & 15306 & 15097 & 14971 & \\
\hline & 14912 & $14953(17)$ & & & & 15102 & 14959 & \\
\hline \multirow{4}{*}{$\frac{3}{2}^{+}$} & 14468 & $14371(12)$ & 14396 & 14347 & 14496 & 14834 & 14432 & 14370 \\
\hline & 14815 & $14840(14)$ & 14805 & 14832 & 15154 & 15089 & 14848 & 14980 \\
\hline & 14893 & $14958(18)$ & 14894 & 14896 & 15300 & & 14975 & \\
\hline & 14905 & $15005(20)$ & & & & & 15016 & \\
\hline \multirow{2}{*}{$\frac{5}{2}^{+}$} & 14895 & 14964(18) & 14894 & 14896 & 15293 & 15101 & 14981 & \\
\hline & 14907 & $15007(20)$ & & & & 15109 & 15022 & \\
\hline$\frac{7}{2}^{+}$ & 14909 & $14969(17)$ & 14894 & 14896 & 15286 & 15101 & 14988 & \\
\hline \multirow{2}{*}{$\frac{1}{2}-$} & 14698 & $14706(9)$ & 14688 & 14645 & 14944 & 14975 & 14773 & \\
\hline & 14991 & & 15016 & & & & & \\
\hline \multirow{2}{*}{$\frac{3}{2}-$} & 14702 & $14714(9)$ & 14688 & 14645 & 14937 & 14976 & 14779 & 14771 \\
\hline & 14922 & & 15016 & & & & & \\
\hline$\frac{5}{2}-$ & 15081 & & 15038 & & 14931 & & & \\
\hline$\frac{7}{2}-$ & 15082 & & & & 15641 & & & \\
\hline
\end{tabular}

TABLE VIII. Comparison with previous theoretical predictions for the masses of the $\Omega_{c c b}$ states.

\begin{tabular}{|c|c|c|c|c|c|c|c|c|}
\hline$J^{P}$ & Our $(\mathrm{MeV})$ & [25] (MeV) & [26] $(\mathrm{MeV})$ & [19] (MeV) & [17] (MeV) & [27] (MeV) & [21] (MeV) & [23] (MeV) \\
\hline \multirow[t]{3}{*}{$\frac{1}{2}^{+}$} & 7984 & $8005(17)$ & $8007(29)$ & 8004 & 8301 & 8005 & 8245 & 7867 \\
\hline & 8361 & & & 8455 & 8647 & 8621 & 8537 & 8337 \\
\hline & 8405 & & & 8536 & & 8848 & & \\
\hline \multirow{3}{*}{$\frac{3}{2}+$} & 7999 & $8026(18)$ & $8037(29)$ & 8023 & 8301 & 8049 & 8265 & 7963 \\
\hline & 8366 & & & 8468 & 8600 & 8637 & 8553 & 8427 \\
\hline & 8412 & & & 8536 & 8647 & 8831 & & \\
\hline \multirow{2}{*}{$\frac{5}{2}^{+}$} & 8473 & & & 8536 & 8647 & 8808 & 8568 & \\
\hline & 8476 & & & & & & 8571 & \\
\hline \multirow[t]{2}{*}{$\frac{7}{2}^{+}$} & 8473 & & & 8538 & 8647 & 8780 & 8568 & \\
\hline & 8538 & & & & & & 8653 & \\
\hline \multirow[t]{2}{*}{$\frac{1}{2}^{-}$} & 8250 & & & 8306 & 8491 & 8400 & 8418 & 8164 \\
\hline & 8266 & & & & & & 8422 & \\
\hline \multirow[t]{2}{*}{$\frac{3}{2}-$} & 8262 & & & 8306 & 8491 & 8383 & 8420 & 8275 \\
\hline & 8268 & & & & & & 8422 & \\
\hline$\frac{5}{2}-$ & 8267 & & & 8311 & 8491 & 8365 & 8432 & \\
\hline
\end{tabular}

fields in Refs. [18,24-26]. Our predictions for the masses of the ground and excited states of the $\Omega_{c c c}$ baryon are lower than lattice [18] results by about 50-150 MeV; however, the structure of our excited spectrum is close to the lattice one. For the $\Omega_{b b b}$ baryon, the agreement of our predictions with lattice results [24] is even better. Masses of only ground states $1 / 2^{+}$and $3 / 2^{+}$of $\Omega_{c c b}$ and $\Omega_{c b b}$ baryons were calculated on the lattice $[25,26]$. They agree well with our results. The constituent quark model, which employs the Gaussian expansion method and the variational principle to solve the nonrelativistic three-body problem, was used in Ref. [19] to compute the mass spectra of triply heavy baryons. The renormalization group procedure for effective particles was applied for studying baryons with heavy quarks in Ref. [17]. The hypercentral constituent quark model was employed in Refs. [20,27,28]. For the calculation of the triply heavy baryon masses, Refs. [21,22] used the nonrelativistic quark model with the harmonic oscillator wave functions and perturbative account of the relativistic corrections. The relativistic Faddeev equation with the rainbow-ladder truncated kernel was employed in Ref. [23].

To demonstrate the necessity of the relativistic treatment of the triply heavy baryons, we calculated the values of 
TABLE IX. Comparison with previous theoretical predictions for the masses of the $\Omega_{c b b}$ states.

\begin{tabular}{|c|c|c|c|c|c|c|c|c|}
\hline$J^{P}$ & Our $(\mathrm{MeV})$ & [25] $(\mathrm{MeV})$ & [26] $(\mathrm{MeV})$ & [19] $(\mathrm{MeV})$ & [17] $(\mathrm{MeV})$ & [28] $(\mathrm{MeV})$ & [21] $(\mathrm{MeV})$ & [23] $(\mathrm{MeV})$ \\
\hline \multirow[t]{3}{*}{$\frac{1}{2}+$} & 11198 & $11194(17)$ & $11195(28)$ & 11200 & 11218 & 11231 & 11535 & 11077 \\
\hline & 11507 & & & 11607 & 11585 & 11757 & 11787 & 11603 \\
\hline & 11622 & & & 11677 & 11626 & 11934 & & \\
\hline \multirow[t]{3}{*}{$\frac{3}{2}^{+}$} & 11217 & 11211(18) & $11229(28)$ & 11221 & 11218 & 11296 & 11554 & 11167 \\
\hline & 11515 & & & 11622 & 11585 & 11779 & 11798 & 11703 \\
\hline & 11629 & & & 11677 & 11626 & 11928 & & \\
\hline \multirow{2}{*}{$\frac{5}{2}+$} & 11632 & & & 11677 & 11626 & 11919 & 11823 & \\
\hline & 11715 & & & & & & 11831 & \\
\hline$\frac{7}{2}^{+}$ & 11635 & & & 11688 & 11626 & 11909 & 11810 & \\
\hline \multirow[t]{2}{*}{$\frac{1}{2}-$} & 11414 & & & 11482 & 11438 & 11573 & 11710 & 11413 \\
\hline & 11506 & & & & & & 11757 & \\
\hline \multirow[t]{2}{*}{$\frac{3}{2}-$} & 11424 & & & 11482 & 11438 & 11566 & 11711 & 11523 \\
\hline & 11535 & & & & & & 11759 & \\
\hline$\frac{5}{2}-$ & 11543 & & & 11569 & 11601 & 11558 & 11762 & \\
\hline
\end{tabular}

$\sqrt{\left\langle v^{2} / c^{2}\right\rangle}$ using obtained triply heavy baryon wave functions. For the ground state of the $\Omega_{c c c}$ baryon, we found the above values of 0.51 and 0.28 for the charm quark and doubly charmed diquark, respectively. For the $\Omega_{b b b}$ baryon, the corresponding values are 0.29 and 0.14 , for the $\Omega_{c c b}$ are 0.20 and 0.30 , and for the $\Omega_{c b b} 0.58$ and 0.10 . For the excited states, these values are even larger. As was expected, the velocities of quarks in these baryons are close to velocities of heavy quarks in quarkonia. We see that even for the heaviest triply bottom baryons relativistic effects are important. They produce additional shifts and splittings of the levels. This calculation demonstrates the need for a relativistic treatment of triply heavy baryons.

\section{CONCLUSIONS}

In this paper, we applied the relativistic quark model based on the quasipotential approach in QCD for the calculation of the mass spectra of triply heavy baryons. The relativistic quark-diquark approximation was used to reduce a very complicated relativistic three-body problem for the subsequent solution of two more simple two-body problems: first calculation of the diquark properties and then considering baryons as a quark-diquark bound system. Such an approach was previously successfully applied for the calculation of the masses of the ground and excited states of doubly heavy [14], heavy [13], and strange baryons [15]. It is important to emphasize that all parameters of the model were kept fixed from the previous calculations, and no new parameters were introduced. We assumed that two identical heavy quarks form a doubly heavy diquark. Masses and wave functions of the ground and excited states of such diquarks were calculated. The internal structure of the diquark was taken into account by the form factor of the diquark-gluon interaction which was calculated as the overlap integral of the diquark wave functions. The internal structure of the diquark was found to be very important for obtaining the correct prediction for the mass of the doubly heavy baryon $\Xi_{c c}$ [14]. It also allows us to obtain local completely relativistic quark-diquark quasipotential without fictitious singularities.

We solved numerically the corresponding quasipotential equation and obtained the masses of the ground and excited states of $\Omega_{c c c}, \Omega_{b b b}, \Omega_{c c b}$, and $\Omega_{c b b}$ baryons. Excited states with total spin up to $J=7 / 2$ both with positive and negative parities were considered. The calculated masses were compared with previous lattice QCD [18,24-26] and quark model calculations. Reasonable agreement with lattice results was found.

\section{ACKNOWLEDGMENTS}

The authors are grateful to D. Ebert, J. Körner, and A. Martynenko for useful discussions. 
[1] Y. R. Liu, H. X. Chen, W. Chen, X. Liu, and S. L. Zhu, Pentaquark and Tetraquark states, Prog. Part. Nucl. Phys. 107, 237 (2019).

[2] N. Brambilla, S. Eidelman, C. Hanhart, A. Nefediev, C. P. Shen, C. E. Thomas, A. Vairo, and C. Z. Yuan, The $X Y Z$ states: Experimental and theoretical status and perspectives, Phys. Rep. 873, 1 (2020).

[3] R. N. Faustov, V. O. Galkin, and E. M. Savchenko, Heavy tetraquarks in the relativistic quark model, Universe 7, 94 (2021).

[4] R. Aaij et al. (LHCb Collaboration), Observation of the doubly charmed baryon $\Xi_{c c}^{++}$, Phys. Rev. Lett. 119, 112001 (2017).

[5] R. Aaij et al. (LHCb Collaboration), Observation of structure in the $J / \psi$-pair mass spectrum, Sci. Bull. 65, 1983 (2020).

[6] R. Aaij et al. (LHCb Collaboration), Observation of an exotic narrow doubly charmed tetraquark, arXiv:2109.01038; Study of the doubly charmed tetraquark $T_{c c}^{+}$, arXiv:2109.01056.

[7] A. Tumasyan et al. (CMS Collaboration), Observation of triple $\mathrm{J} / \psi$ meson production in proton-proton collisions at $\sqrt{s}=13 \mathrm{TeV}$, arXiv:2111.05370.

[8] Y.Q. Chen and S.Z. Wu, Production of triply heavy baryons at LHC, J. High Energy Phys. 08 (2011) 144; 09 (2011) 89(E).

[9] H. He, Y. Liu, and P. Zhuang, $\Omega_{c c c}$ production in high energy nuclear collisions, Phys. Lett. B 746, 59 (2015).

[10] D. Ebert, V. O. Galkin, and R. N. Faustov, Properties of heavy quarkonia and $B_{c}$ mesons in the relativistic quark model, Phys. Rev. D 67, 014027 (2003); Spectroscopy and Regge trajectories of heavy quarkonia and $B_{c}$ mesons, Eur. Phys. J. C 71, 1825 (2011).

[11] D. Ebert, R. N. Faustov, and V. O. Galkin, Mass spectra and Regge trajectories of light mesons in the relativistic quark model, Phys. Rev. D 79, 114029 (2009); Heavy-light meson spectroscopy and Regge trajectories in the relativistic quark model, Eur. Phys. J. C 66, 197 (2010).

[12] D. Ebert, R. N. Faustov, and V. O. Galkin, Masses of heavy baryons in the relativistic quark model, Phys. Rev. D 72, 034026 (2005); Masses of excited heavy baryons in the relativistic quark model, Phys. Lett. B 659, 612 (2008).

[13] D. Ebert, R. N. Faustov, and V. O. Galkin, Spectroscopy and Regge trajectories of heavy baryons in the relativistic quarkdiquark picture, Phys. Rev. D 84, 014025 (2011).

[14] D. Ebert, R. N. Faustov, V. O. Galkin, and A. P. Martynenko, Mass spectra of doubly heavy baryons in the relativistic quark model, Phys. Rev. D 66, 014008 (2002).

[15] R. N. Faustov and V. O. Galkin, Strange baryon spectroscopy in the relativistic quark model, Phys. Rev. D 92, 054005 (2015).

[16] R. N. Faustov, V. O. Galkin, and E. M. Savchenko, Masses of the $Q Q \bar{Q} \bar{Q}$ tetraquarks in the relativistic diquarkantidiquark picture, Phys. Rev. D 102, 114030 (2020).

[17] K. Serafin, M. Gómez-Rocha, J. More, and S. D. Głazek, Approximate Hamiltonian for baryons in heavy-flavor QCD, Eur. Phys. J. C 78, 964 (2018).

[18] M. Padmanath, R. G. Edwards, N. Mathur, and M. Peardon, Spectroscopy of triply-charmed baryons from lattice QCD, Phys. Rev. D 90, 074504 (2014).
[19] G. Yang, J. Ping, P. G. Ortega, and J. Segovia, Triply heavy baryons in the constituent quark model, Chin. Phys. C 44, 023102 (2020).

[20] Z. Shah and A. K. Rai, Masses and Regge trajectories of triply heavy $\Omega_{c c c}$ and $\Omega_{b b b}$ baryons, Eur. Phys. J. A 53, 195 (2017).

[21] W. Roberts and M. Pervin, Heavy baryons in a quark model, Int. J. Mod. Phys. A 23, 2817 (2008).

[22] M. S. Liu, Q. F. Lü, and X. H. Zhong, Triply charmed and bottom baryons in a constituent quark model, Phys. Rev. D 101, 074031 (2020).

[23] S. X. Qin, C. D. Roberts, and S. M. Schmidt, Spectrum of light- and heavy-baryons, Few-Body Syst. 60, 26 (2019).

[24] S. Meinel, Excited-state spectroscopy of triply-bottom baryons from lattice QCD, Phys. Rev. D 85, 114510 (2012).

[25] N. Mathur, M. Padmanath, and S. Mondal, Precise Predictions of Charmed-Bottom Hadrons from Lattice QCD, Phys. Rev. Lett. 121, 202002 (2018).

[26] Z.S. Brown, W. Detmold, S. Meinel, and K. Orginos, Charmed bottom baryon spectroscopy from lattice QCD, Phys. Rev. D 90, 094507 (2014).

[27] Z. Shah and A. Kumar Rai, Spectroscopy of the $\Omega_{c c b}$ baryon in the hypercentral constituent quark model, Chin. Phys. C 42, 053101 (2018).

[28] Z. Shah and A. K. Rai, Ground and excited state masses of the $\Omega_{b b c}$ baryon, Few-Body Syst. 59, 76 (2018).

[29] K. U. Can, G. Erkol, M. Oka, and T. T. Takahashi, Look inside charmed-strange baryons from lattice QCD, Phys. Rev. D 92, 114515 (2015).

[30] Y. Namekawa et al. (PACS-CS Collaboration), Charmed baryons at the physical point in $2+1$ flavor lattice QCD, Phys. Rev. D 87, 094512 (2013).

[31] R. A. Briceno, H. W. Lin, and D. R. Bolton, Charmedbaryon spectroscopy from lattice QCD with $N_{f}=2+1+$ 1 flavors, Phys. Rev. D 86, 094504 (2012).

[32] C. Alexandrou, V. Drach, K. Jansen, C. Kallidonis, and G. Koutsou, Baryon spectrum with $N_{f}=2+1+1$ twisted mass fermions, Phys. Rev. D 90, 074501 (2014).

[33] J. Vijande, A. Valcarce, and H. Garcilazo, Constituent-quark model description of triply heavy baryon nonperturbative lattice QCD data, Phys. Rev. D 91, 054011 (2015).

[34] B. Silvestre-Brac, Spectrum and static properties of heavy baryons, Few-Body Syst. 20, 1 (1996).

[35] P. Hasenfratz, R. R. Horgan, J. Kuti, and J. M. Richard, Heavy baryon spectroscopy in the QCD bag model, Phys. Lett. 94B, 401 (1980).

[36] A. P. Martynenko, Ground-state triply and doubly heavy baryons in a relativistic three-quark model, Phys. Lett. B 663, 317 (2008).

[37] J. R. Zhang and M. Q. Huang, Deciphering triply heavy baryons in terms of QCD sum rules, Phys. Lett. B 674, 28 (2009).

[38] T. M. Aliev, K. Azizi, and M. Savci, Properties of triply heavy spin-3/2 baryons, J. Phys. G 41, 065003 (2014).

[39] Z. G. Wang, Analysis of the triply heavy baryon states with QCD sum rules, Commun. Theor. Phys. 58, 723 (2012).

[40] S. Migura, D. Merten, B. Metsch, and H. R. Petry, Charmed baryons in a relativistic quark model, Eur. Phys. J. A 28, 41 (2006). 
[41] M. Radin, S. Babaghodrat, and M. Monemzadeh, Estimation of heavy baryon masses $\Omega_{c c c}^{++}$and $\Omega_{b b b}^{-}$by solving the Faddeev equation in a three-dimensional approach, Phys. Rev. D 90, 047701 (2014).

[42] K. Thakkar, A. Majethiya, and P. C. Vinodkumar, Magnetic moments of baryons containing all heavy quarks in the quark-diquark model, Eur. Phys. J. Plus 131, 339 (2016).

[43] P. L. Yin, C. Chen, G. Krein, C. D. Roberts, J. Segovia, and $\mathrm{S} . \mathrm{S}$. $\mathrm{Xu}$, Masses of ground-state mesons and baryons, including those with heavy quarks, Phys. Rev. D 100, 034008 (2019).

[44] Y. Jia, Variational study of weakly coupled triply heavy baryons, J. High Energy Phys. 10 (2006) 073.

[45] K. W. Wei, B. Chen, and X. H. Guo, Masses of doubly and triply charmed baryons, Phys. Rev. D 92, 076008 (2015).

[46] K. W. Wei, B. Chen, N. Liu, Q. Q. Wang, and X. H. Guo, Spectroscopy of singly, doubly, and triply bottom baryons, Phys. Rev. D 95, 116005 (2017). 